Algebraic $8 \mathcal{G}$ Geometric $\mathcal{T}_{\text {opology }}$

Volume 5 (2005) 769-784

Published: 23 July 2005

ATG

\title{
Pinwheels and bypasses
}

\author{
KO HONDA \\ William H. KAZEZ \\ Gordana Matić
}

\begin{abstract}
We give a necessary and sufficient condition for the addition of a collection of disjoint bypasses to a convex surface to be universally tight - namely the nonexistence of a polygonal region which we call a virtual pinwheel.
\end{abstract}

AMS Classification 57M50; 53C15

Keywords Tight, contact structure, bypass, pinwheel, convex surface

\section{Introduction}

In this paper we assume that our 3-manifolds are oriented and our contact structures cooriented. Let $\Sigma$ be a convex surface, i.e., it admits a $[-\varepsilon, \varepsilon]$-invariant contact neighborhood $\Sigma \times[-\varepsilon, \varepsilon]$, where $\Sigma=\Sigma \times\{0\}$. We do not assume that a convex surface $\Sigma$ is closed or compact, unless specified. According to a theorem of Giroux [4, if $\Sigma \neq S^{2}$ is closed or compact with Legendrian boundary, then $\Sigma$ has a tight neighborhood if and only if its dividing set $\Gamma_{\Sigma}$ has no homotopically trivial closed curves. (In the case when $\Sigma$ is not necessarily compact, $\Sigma$ has a tight neighborhood if $\Gamma_{\Sigma}$ has no homotopically trivial dividing curves, although the converse is not always true.) In this paper we study the following:

Question 1.1 Suppose we attach a family of bypasses $\mathcal{B}=\left\{\mathcal{B}_{\alpha}\right\}_{\alpha \in A}$ along a disjoint family of Legendrian $\operatorname{arcs} \mathcal{C}=\left\{\delta_{\alpha}\right\}_{\alpha \in A}$ to a product tight contact structure on $\Sigma \times[-\varepsilon, \varepsilon]$. When is the resulting contact manifold tight?

A closed Legendrian arc $\delta_{\alpha}$, along which a bypass $\mathcal{B}_{\alpha}$ for $\Sigma$ is attached, is called a Legendrian arc of attachment. Every arc of attachment begins and ends on $\Gamma_{\Sigma}$ and has three intersection points with $\Gamma_{\Sigma}$, all of which are transverse. In this paper all bypasses are assumed to be attached "from the front", i.e., attached along $\Sigma \times\{\varepsilon\}$ from the exterior of $\Sigma \times[-\varepsilon, \varepsilon]$, and all arcs of attachment are 
assumed to be embedded, i.e., there are no "singular bypasses". Recall from [5] that attaching $\mathcal{B}_{\alpha}$ from the front and isotoping the surface $\Sigma$ across the bypass is locally given by Figure 11 Denote by $(\Sigma, \mathcal{C})$ the contact manifold $(\Sigma \times[-\varepsilon, \varepsilon]) \cup\left(\cup_{\alpha} N\left(\mathcal{B}_{\alpha}\right)\right)$, where $N\left(\mathcal{B}_{\alpha}\right)$ is an invariant neighborhood of $\mathcal{B}_{\alpha}$.

We will show that the key indicator of overtwistedness in the resulting contact manifold $(\Sigma, \mathcal{C})$ is a polygonal region in $\Sigma$ called a pinwheel. First consider an embedded polygonal region $P$ in $\Sigma$ whose boundary consists of $2 k$ consecutive sides $\gamma_{1}, \alpha_{1}, \gamma_{2}, \alpha_{2}, \ldots, \gamma_{k}, \alpha_{k}$ in counterclockwise order, where each $\gamma_{i}$ is a subarc of $\Gamma_{\Sigma}$ and each $\alpha_{i}$ is a subarc of a Legendrian arc of attachment $\delta_{i} \in \mathcal{C}$. Here $k \geq 1$. In this paper, when we refer to a "polygon", we will tacitly assume that it is an embedded polygonal region of the type just described. Now orient the sides using the boundary orientation of $P$. A pinwheel is a special type of polygon $P$, where, for each $i=1, \ldots, k, \delta_{i}$ extends past the final point of $\alpha_{i}$ (not past the initial point) and does not reintersect $P$. (If $k>1$, then this is equivalent to asking $\delta_{i}$ to extend past $\gamma_{i+1}$, where $i$ is considered modulo $k$.) Figure 3 gives an example of a pinwheel.

It is easy to see that, if $\Sigma$ is closed or compact with Legendrian boundary, then the addition of bypasses along all the arcs of attachment of a pinwheel produces an overtwisted disk manifested by a contractible curve in the resulting dividing set. Hence, the nonexistence of pinwheels is a necessary condition for the new contact structure to be tight. Essentially, we are asking that no closed, homotopically trivial curves be created when some or all of the bypasses are attached. We will prove that the nonexistence of pinwheels is a sufficient condition as well, if $\Sigma$ is a disk with Legendrian boundary.

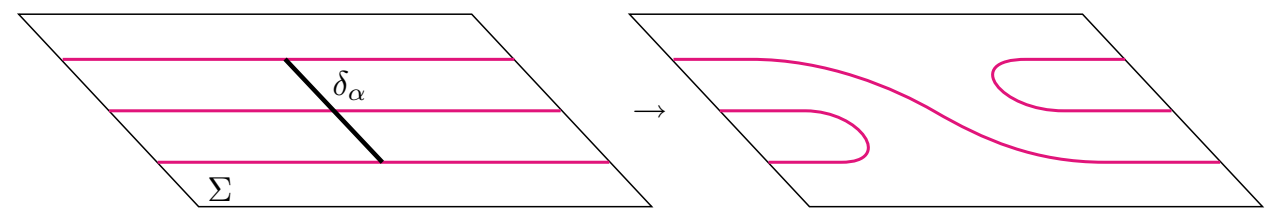

Figure 1: Adding a bypass

Theorem 1.2 Let $\Sigma$ be a convex disk with Legendrian boundary and with a tight neighborhood, and let $\mathcal{C}$ be a finite, disjoint collection of bypass arcs of attachment on $\Sigma$. Denote by $(\Sigma, \mathcal{C})$ the contact structure on $\Sigma \times I$ obtained by attaching to the product contact neighborhood of $\Sigma$ bypasses along all the arcs in $\mathcal{C}$. Then $(\Sigma, \mathcal{C})$ is tight if and only if there are no pinwheels in $\Sigma$.

If a compact convex surface $\Sigma$ has $\pi_{1}(\Sigma) \neq 0$, then Theorem 1.2 is modified to allow virtual pinwheels - a virtual pinwheel is an embedded polygon $P$ which 
becomes a pinwheel in some finite cover of $\Sigma$. In other words, since the fundamental group of every compact surface is residually finite, a virtual pinwheel $P$ is either already a pinwheel or the arcs of attachment $\delta_{i}$ which comprise its sides may extend beyond the polygon, encircle a nontrivial element in $\pi_{1}(\Sigma, P)$ and return to the polygon. Figure 2 gives examples of arcs of attachment which we will show result in overtwisted contact structures. The figure to the left is a pinwheel, the one to the right is an example of a virtual pinwheel.
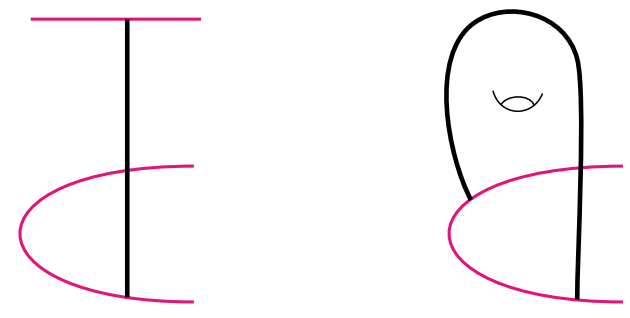

Figure 2: A pinwheel and a virtual pinwheel

Theorem 1.3 Let $\Sigma \neq S^{2}$ be a convex surface which is closed or compact with Legendrian boundary and which has a tight neighborhood, and let $\mathcal{C}$ be a finite disjoint collection of arcs of attachment. Then the following are equivalent:

(1) $(\Sigma, \mathcal{C})$ is universally tight.

(2) There are no virtual pinwheels in $\Sigma$.

Remark 1.4 A pinwheel $P$ may nontrivially intersect arcs of $\mathcal{C}$ in its interior. Any such arc $\delta^{\prime}$ would cut $P$ into two polygons, and one of the two polygons $P^{\prime}$ will satisfy the definition of a pinwheel, with the possible exception of the condition that $\delta^{\prime}$ not reintersect $P^{\prime}$. (If $\delta^{\prime}$ does not reintersect $P^{\prime}$, then we can shrink $P$ to $P^{\prime}$.)

\section{Proof of Theorem 1.2}

Let $\mathcal{C}$ be the collection of arcs of attachment and $\mathcal{B}_{\delta}$ be the bypass corresponding to $\delta \in \mathcal{C}$.

The "only if" direction is immediate. If there is a pinwheel $P$, then let $\alpha_{i}$, $i=1, \ldots, k$, be the sides of $P$ which are subarcs of $\delta_{i} \in \mathcal{C}$. Then attaching all the bypasses $\mathcal{B}_{\delta_{i}}$ creates a closed homotopically trivial curve, and hence an overtwisted disk. We can think of this disk as living at some intermediate level 


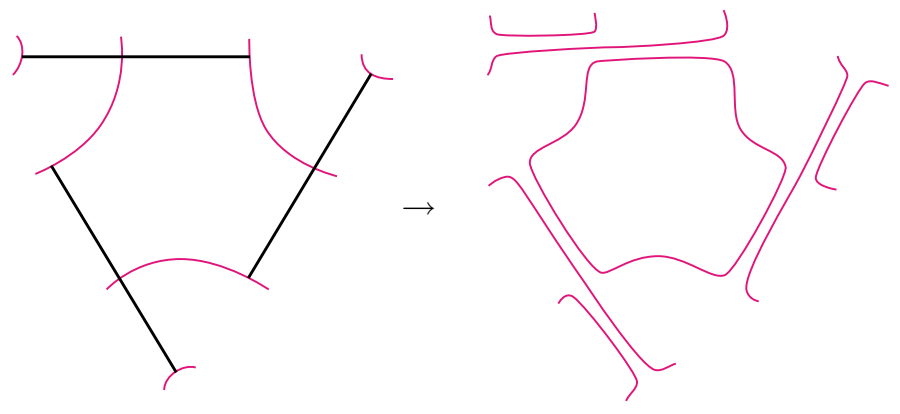

Figure 3: Attaching the bypasses around a pinwheel

$\Sigma \times\{t\}$ in the contact structure on $\Sigma \times I$ obtained by attaching all the bypasses determined by $\operatorname{arcs}$ in $\mathcal{C}$. See Figure 3 ,

Remark 2.1 The arcs of attachment may be trivial arcs of attachment. For example, if only one of them is attached, $\Gamma_{\Sigma}$ does not change. However, a "trivial arc" $\delta_{j}$, after it is attached, may affect the positions of the other arcs of attachment, if they intersect the subarc $\gamma \subset \Gamma_{\Sigma}$ which forms a polygon together with a subarc of $\delta_{j}$. Therefore, "trivial" arcs are not necessarily genuinely trivial as part of a family.

Remark 2.2 It is crucial that an $\operatorname{arc} \delta_{i}$ in the definition of a pinwheel not return to $P$. For example, if some $\delta_{j}$ returns to $\gamma_{j+1}$, then no overtwisted disk appears in a neighborhood of the original $P$, after all the bypasses $\mathcal{B}_{\delta_{i}}$ are attached.

We now prove the "if" part, namely if there are no pinwheels, then the attachment of $\mathcal{C}$ onto the convex disk $\Sigma$ is tight. In fact, we prove the following stronger result:

Theorem 2.3 Let $\Sigma$ be a convex plane whose dividing set $\Gamma_{\Sigma}$ has no connected components which are closed curves. If $\mathcal{C}$ is a locally finite, disjoint collection of bypass arcs of attachment on $\Sigma$, and $\Sigma$ has no pinwheels, then $(\Sigma, \mathcal{C})$ is tight.

Reduction of Theorem 2.3 to Theorem 1.2 Since any overtwisted disk will live in a compact region of $\Sigma \times[-\varepsilon, \varepsilon]$, we use an exhaustion argument to reduce to the situation where we have a closed disk $D$ with Legendrian boundary, and $\mathcal{C}$ is a finite collection of arcs of attachment which avoid $\partial D$. 
There is actually one subtlety here when we try to use the Legendrian Realization Principle (LeRP) on a noncompact $\Sigma$ to obtain Legendrian boundary for $D$ - it is that there is no bound on the distance (with respect to any complete metric on $\Sigma$ ) traveled by $\partial D$ during the isotopy given in the proof of the Giroux Flexibility Theorem. Hence we take a different approach, namely exhausting $\Sigma$ by convex disks $D_{i}$ where $\partial D_{i}$ is not necessarily Legendrian, and then extending $D_{i}$ to a convex disk $D_{i}^{\prime}$ with Legendrian boundary and without pinwheels.

Let $D_{1} \subset D_{2} \subset \ldots$ be such an exhaustion of $\Sigma$, with the additional property that $\partial D_{i} \pitchfork \Gamma_{\Sigma}$ and moreover at each intersection point $x$ the characteristic foliation and $\partial D_{i}$ agree on some small interval around $x$. Consider a rectangle $R=[0, n] \times[0,1]$ with coordinates $(x, y)$. Let $s$ be an arc on $\partial D_{i}$ between two consecutive intersections of $\Gamma_{\Sigma} \cap \partial D_{i}$. Take a diffeomorphism which takes $s$ to $x=0$; let $\xi$ be the induced contact structure in a neighborhood of $x=0$. It is easy to extend $\xi$ to (a neighborhood of) $y=0$ and $y=1$ so that they become dividing curves. Now the question is to extend $\xi$ to all of $R$ so that $x=n$ is Legendrian. Let $R^{\prime}=[0, n] \times\left[\varepsilon^{\prime}, 1-\varepsilon^{\prime}\right] \subset R$ be a slightly smaller rectangle. We write the sought-after invariant contact form on $R^{\prime} \times[-\varepsilon, \varepsilon]$ as $\alpha=d t+\beta$, where $t$ is the coordinate for $[-\varepsilon, \varepsilon], \beta$ is a form on $R^{\prime}$ which does not depend on $t$, and $d \beta$ is an area form on $R^{\prime}$. Provided $n$ is sufficiently large, $\int_{\partial R^{\prime}} \beta$ will be positive, regardless of $\beta$ on $x=0$. Let $\omega$ be an area form on $R^{\prime}$ which agrees with $d \beta$ on $\partial R^{\prime}$ and satisfies $\int_{R^{\prime}} \omega=\int_{\partial R^{\prime}} \beta$. Extend $\left.\beta\right|_{\partial R^{\prime}}$ to any 1-form $\beta^{\prime}$ on $R^{\prime}$ (not necessarily the primitive of an area form). Since $d \beta^{\prime}$ agrees with $\omega$ on $\partial R^{\prime}$, consider $\omega-d \beta^{\prime} . \int_{R^{\prime}} \omega-d \beta^{\prime}=0$ and $\omega-d \beta^{\prime}=0$ on $\partial R^{\prime}$, so by the Poincaré lemma there is a 1 -form $\beta^{\prime \prime}$ with $\left.\beta^{\prime \prime}\right|_{\partial R^{\prime}}=0$, so that $\omega-d \beta^{\prime}=d \beta^{\prime \prime}$. Therefore, the desired $\beta$ on $R^{\prime}$ is $\beta^{\prime}+\beta^{\prime \prime}$. Since there are only finitely many components of $\Gamma_{\Sigma} \cap D_{i}$, we obtain $D_{i}^{\prime}$ by attaching finitely many rectangles of the type described above.

Let us now consider the pair $(D, \mathcal{C})$ consisting of a convex disk $D$ with Legendrian boundary (and dividing set $\Gamma_{D}$ ) and a finite collection $\mathcal{C}$ of Legendrian arcs of attachment for $D$. We now prove the following:

Proposition 2.4 If $(D, \mathcal{C})$ has no pinwheels, then $(D, \mathcal{C})$ is tight.

Proof The idea is to induct on the complexity of the situation. Here, the complexity $c(D, \mathcal{C})$ of $(D, \mathcal{C})$ is given by $c(D, \mathcal{C})=\# \Gamma_{D}+\# \mathcal{C}$, where $\# \Gamma_{D}$ is the number of connected components of $\Gamma_{D}$ and $\# \mathcal{C}$ is the number of bypass arcs in $\mathcal{C}$. Given $(D, \mathcal{C})$ we will find a pair $\left(D^{\prime}, \mathcal{C}^{\prime}\right)$ of lower complexity, where 
$(D, \mathcal{C})$ is tight if $\left(D^{\prime}, \mathcal{C}^{\prime}\right)$ is tight, and $\left(D^{\prime}, \mathcal{C}^{\prime}\right)$ has no pinwheels if $(D, \mathcal{C})$ has no pinwheels.

The proof will proceed by showing that there are three operations, which we call $\mathrm{A}, \mathrm{B}$, and $\mathrm{C}$, one of which can always be performed to reduce the complexity until there are no bypasses left. Operation A removes (unnecessary) isolated $\partial$-parallel arcs. If isolated $\partial$-parallel arcs do not exist, we apply one of Operations $\mathrm{B}$ and $\mathrm{C}$. If there is a trivial bypass in $\mathcal{C}$, Operation $\mathrm{B}$ removes an "innermost" trivial bypass, i.e., we show that performing the bypass attachment gives a configuration with lower complexity which is tight if and only if the original configuration was tight. Otherwise, Operation C removes an "outermost" nontrivial bypass by embedding the configuration into one of lower complexity. Since each step is strictly complexity-decreasing, and we can always do at least one of them, we can always perform the inductive step. This will prove the proposition and the theorem.

\subsection{Operation A: isolated $\partial$-parallel arc}

Suppose $\Gamma_{D}$ has a $\partial$-parallel arc $\gamma$ which does not intersect any component of $\mathcal{C}$. (Recall that arcs of attachment are assumed to be closed and hence no component of $\mathcal{C}$ begins or ends on $\gamma$.) We then extend $D$ to $D^{\prime}$ so that $t b\left(D^{\prime}\right)=$ $t b(D)+1$ and $\Gamma_{D^{\prime}}$ is obtained from $\Gamma_{D}$ by connecting one of the endpoints of $\gamma$ to a neighboring endpoint of another arc in $\Gamma_{D}$. (See Figure 4.) It is clear that since the configuration $(D, \mathcal{C})$ can be embedded into the configuration $\left(D^{\prime}, \mathcal{C}^{\prime}=\mathcal{C}\right)$, and vice versa, $(D, \mathcal{C})$ tight is equivalent to $\left(D^{\prime}, \mathcal{C}^{\prime}\right)$ tight, and $(D, \mathcal{C})$ having no pinwheels is equivalent to $\left(D^{\prime}, \mathcal{C}^{\prime}\right)$ with no pinwheels.

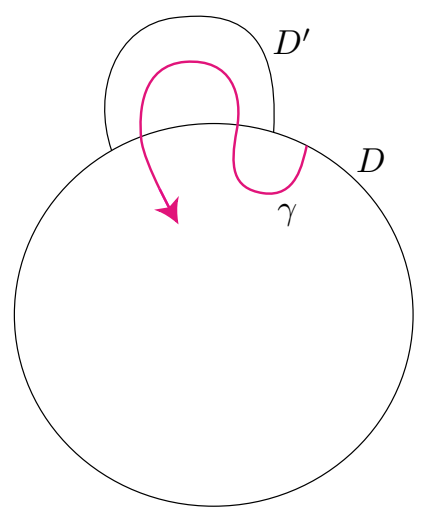

Figure 4: The new $D^{\prime}$ 


\subsection{Operation B: trivial bypasses}

Suppose there is a trivial arc of attachment $\delta$ in $\mathcal{C}$. Let $\gamma$ be the connected component of $\Gamma_{D}$ that $\delta$ intersects at least twice, and let $R$ be a closed half-disk (polygon) whose two sides are a subarc of $\delta$ and a subarc $\gamma_{0}$ of $\gamma$. As shown in Figure 5, we choose $R$ to be such that, with respect to the orientation of $\delta$ induced by $\partial R$, the subarc of $\delta$ contained in $\partial R$ starts at an interior point of $\delta$. If $\mathcal{C}-\{\delta\}$ nontrivially intersects $\operatorname{int}(R)$, then let $\delta^{\prime}$ be an arc of $\mathcal{C}-\{\delta\}$ which is outermost in $R$, i.e., cuts off a subpolygon of $R$ which does not intersect $\mathcal{C}-\{\delta\}$ in its interior. Define $R^{\prime}$ and $\gamma_{0}^{\prime}$ analogously for $\delta^{\prime}$. (Note that $R^{\prime}$ may or may not be a subset of $R$.) We rename $\delta^{\prime}, R^{\prime}$ and $\gamma_{0}^{\prime}$ by omitting primes. Therefore, we may assume that $\delta, \gamma$, and $R$ satisfy the property that $\operatorname{int}(R)$ does not intersect any arc of $\mathcal{C}$, although there may be endpoints of arcs of $\mathcal{C}-\{\delta\}$ along $\gamma_{0}$. By the very definition of $R$, the third point of intersection between $\delta$ and $\Gamma_{D}$ cannot be in $\gamma_{0}$.

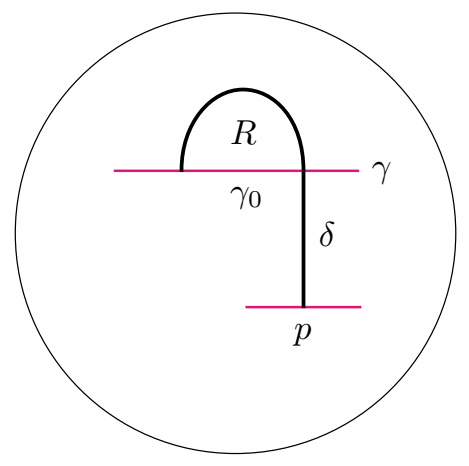

Figure 5: A trivial arc of attachment

Now, let $D=D^{\prime}$ and let $\Gamma_{D^{\prime}}$ be the dividing set obtained from $\Gamma_{D}$ by attaching the bypass $\delta$ (the dividing set is modified in a neighborhood of $\delta$ ). The isotopy type of $\Gamma_{D}$ and $\Gamma_{D^{\prime}}$ are the same. However, $\mathcal{C}^{\prime}$ is identical to $\mathcal{C}-\{\delta\}$ with the following exception: arcs $\delta_{i} \in \mathcal{C}-\{\delta\}$ which ended on $\gamma_{0} \subset \gamma$ now end on (what we may think of as) a small interval of $\Gamma_{D^{\prime}}=\Gamma_{D}$ around $p$. See Figures [6] and 7 which both depict what happens locally near $\delta$. We emphasize that in Figures 6 and 7 the two dividing curves may be part of the same dividing curve.

Claim If $(D, \mathcal{C})$ has no pinwheels then neither does $\left(D^{\prime}, \mathcal{C}^{\prime}\right)$.

Proof For an $\operatorname{arc} \delta_{i}$ in $\mathcal{C}-\{\delta\}$ with an endpoint $q$ on $\gamma_{0}$, let $\delta_{i}^{\prime}$ be its image in $\mathcal{C}^{\prime}$. If a pinwheel $P^{\prime}$ of $D^{\prime}$ has a subarc of $\delta_{i}^{\prime}$ as a side and $q$ as a vertex, it is 
clear that there was a pinwheel $P$ of $D$ which had subarcs of $\delta_{i}$ and $\delta$ as sides. The pinwheels $P$ and $P^{\prime}$ are basically the same region of $D$ - all the sides are the same except that $P$ has two extra vertices, $p$ and $r$, and two extra sides. Here $r$ is the middle intersection point of $\delta$ with $\Gamma_{D}$ as in Figure 6
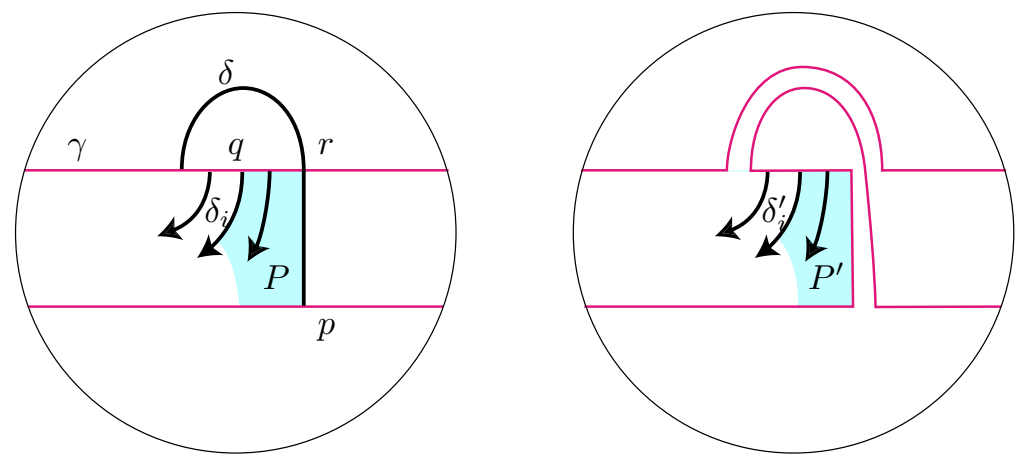

Figure 6: Pinwheels in $D$ and $D^{\prime}$

On the other hand, suppose $P^{\prime}$ is a pinwheel of $D^{\prime}$ which does not involve any subarcs which used to intersect $\gamma_{0}$. Then $P^{\prime}$ must either completely contain or be disjoint from the region $K^{\prime}$ given in Figure 7 . However, apart from $K^{\prime}$ (and the corresponding region $K$ in $D),(D, \mathcal{C}-\{\delta\})$ and $\left(D^{\prime}, \mathcal{C}^{\prime}\right)$ are identical. Hence $P^{\prime}$ must have descended from a pinwheel for $D$.
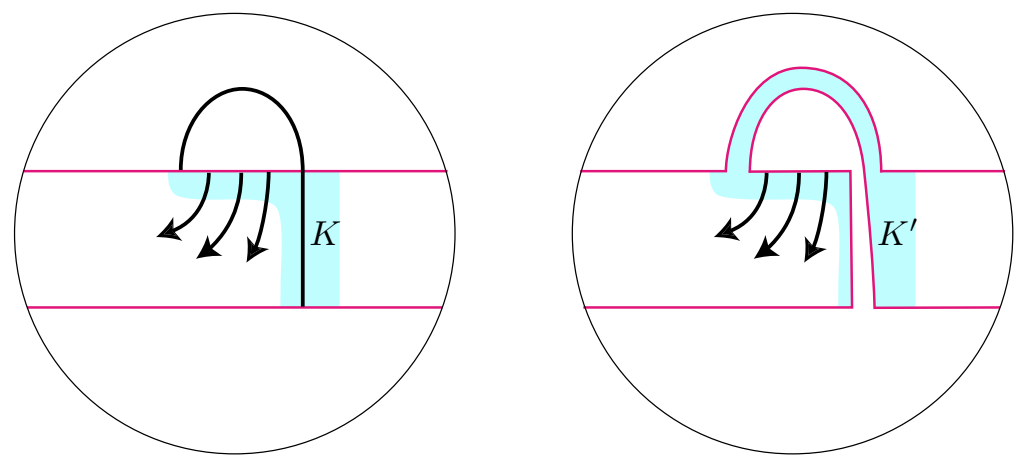

Figure 7: Pinwheels in $D$ and $D^{\prime}$

\subsection{Operation C: outermost nontrivial bypass}

Suppose all the arcs of $\mathcal{C}$ are nontrivial and there are no isolated $\partial$-parallel arcs. Then we have the following: 
Claim There exists an "outermost" arc $\delta$ with the following property: there exists an orientation/parametrization of $\delta$ so it intersects distinct arcs $\gamma_{3}, \gamma_{2}$, $\gamma_{1}$ of $\Gamma_{D}$ in that order, and if $R \subset D$ is the closed region cut off by the subarc $\alpha$ of $\delta$ from $\gamma_{3}$ to $\gamma_{2}$ and subarcs of $\gamma_{2}$ and $\gamma_{3}$ so that the boundary orientation on $\alpha$ induced from $R$ and the orientation from $\delta$ are opposite, then $\operatorname{int}(R)$ does not intersect any other arcs of $\mathcal{C}$.
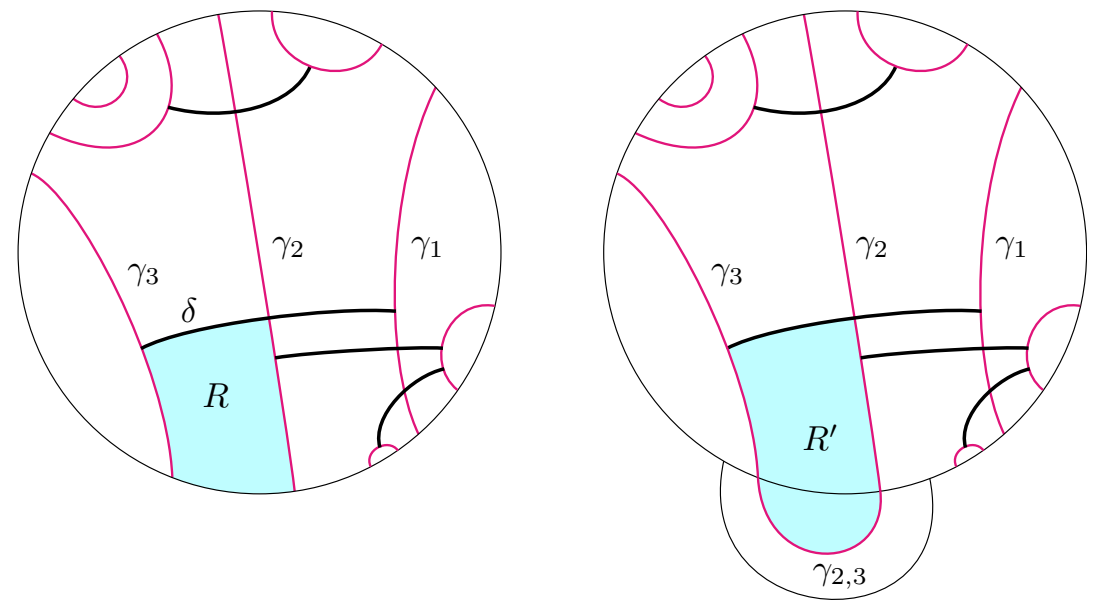

Figure 8: An outermost bypass arc

Proof Let $\gamma$ be a $\partial$-parallel arc of $\Gamma_{D}$. Since there are no isolated $\partial$-parallel arcs or trivial bypasses, $\gamma$ contains an endpoint of at least one arc of attachment in $\mathcal{C}$. Of all such arcs of attachment ending on $\gamma$, choose the "rightmost" one $\delta$, if we represent $D$ as the unit disk, $\gamma$ is in the $x$-axis, and the half-disk cut off by $\gamma$ with no other intersections with $\Gamma_{D}$ is in the lower half-plane. Now orient $\delta$ so that $\gamma_{3}=\gamma$ and denote by $\gamma_{2}$ the next arc in $\Gamma_{D}$ that $\delta$ intersects. Let $R$ be the region bounded by $\gamma_{3}, \delta, \gamma_{2}$ and an arc in the boundary of $D$, so that the boundary orientation of $R$ and the orientation of $\delta$ are opposite. There are no endpoints of arcs of $\mathcal{C}-\{\delta\}$ along $\partial R \cap \gamma_{3}$, but there may certainly be arcs which intersect $\operatorname{int}(R)$ and $\partial R \cap \gamma_{2}$. If there are no $\partial$-parallel arcs in $\operatorname{int}(R)$, then we are done. Otherwise, take the clockwisemost $\partial$-parallel arc $\gamma^{\prime}$ of $\Gamma_{D}$ (along $\partial D$ ) in $\operatorname{int}(R)$, and let $\delta^{\prime}$ be the rightmost arc of $\mathcal{C}$ starting from $\gamma^{\prime}$. Its corresponding region $R^{\prime}$ is strictly contained in $R$; hence if we rename everything by removing primes and reapply the same procedure, then eventually we obtain $\gamma, \delta$, and $R$ so that no $\operatorname{arc}$ of $\mathcal{C}$ intersects $\operatorname{int}(R)$. 
Let $\delta$ be an outermost bypass (in the sense of the previous claim). Then there exists an extension $D^{\prime}$ of $D$, where $t b\left(D^{\prime}\right)=t b(D)+1$ and $\Gamma_{D^{\prime}}$ is obtained from $\Gamma_{D}$ by connecting the endpoints of $\gamma_{2}$ and $\gamma_{3}$ (those which are corners of $R$ ) by an arc. If $\gamma_{2,3}$ is the resulting connected component of $\Gamma_{D^{\prime}}$ which contains $\gamma_{2}$ and $\gamma_{3}$, then $\gamma_{2,3}$ and $\delta$ cobound a disk region $R^{\prime} \subset D^{\prime}$ that contains $R$. Observe that $D^{\prime}$ has a tight neighborhood, i.e., $\Gamma_{D^{\prime}}$ contains no closed loops, because $\gamma_{2}$ and $\gamma_{3}$ were distinct arcs of $\Gamma_{D}$. Now set $\mathcal{C}^{\prime}=\mathcal{C}$. Then $\left(D^{\prime}, \mathcal{C}^{\prime}\right)$ has lower complexity than $(D, \mathcal{C})$. It is clear that $\left(D^{\prime}, \mathcal{C}^{\prime}\right)$ has no pinwheels: any pinwheel $P^{\prime}$ of $\left(D^{\prime}, \mathcal{C}^{\prime}\right)$ is either already a pinwheel in $(D, \mathcal{C})$ or contains $R^{\prime}$. However, any pinwheel that contains $R^{\prime}$ must extend beyond $\delta$, and hence must contain a sub-pinwheel $P$ with a side $\delta$ inherited from $(D, \mathcal{C})$.

\section{Proof of Theorem 1.3}

$(1) \Rightarrow(2)$ is clear. Namely, if there is a virtual pinwheel in $\Sigma$, there is a pinwheel in some finite cover, and hence that cover is overtwisted.

$(2) \Rightarrow(1)$ Assume that, on the contrary, $(\Sigma, \mathcal{C})$ is not universally tight. Let $D$ be a disk in the universal cover such that restriction to $D \times I$ contains the overtwisted disk. We can find a finite cover $\widetilde{\Sigma}$ of $\Sigma$ that contains that disk, and by modifying the characteristic foliation using LeRP if necessary, we can assume that the disk has Legendrian boundary. Then by Theorem 1.2 there is a pinwheel $P$ in $\widetilde{\Sigma}$. We will show that this implies the existence of a virtual pinwheel in $\Sigma$.

Let $\pi: \widetilde{\Sigma} \rightarrow \Sigma$ be the projection map and $\mathcal{P}$ be the set of polygons $R$ of $\Sigma$ which are minimal in the sense that they do not contain smaller subpolygons. Then we can define the weight function $w: \mathcal{P} \rightarrow \mathbb{Z}$, which assigns to each $R \in \mathcal{P}$ the degree of $\pi^{-1}(R) \cap P$ over $R$. We illustrate this definition in Figure9, The shaded area in the left half of the picture is the pinwheel $P$ in $\widetilde{\Sigma}$. The polygonal regions in $\Sigma$ are labeled by integers 0,1 and 2 according to the value that $w$ takes on them. Our sought-after virtual pinwheel $P^{\prime} \subset \Sigma$ is then one connected component of the union of all $R \in \mathcal{P}$ which attain the maximal value of $w$. (In the figure there is only one component.)

First observe that $w$ cannot be locally constant, since $P$ is strictly contained in either the positive region $R_{+}\left(\Gamma_{\widetilde{\Sigma}}\right)$ or the negative region $R_{-}\left(\Gamma_{\widetilde{\Sigma}}\right)$ - for convenience let us suppose it is $R_{+}$. Next we show that the values of $w$ are different for any two polygonal regions $R_{1}$ and $R_{2}$ inside $R_{+}\left(\Gamma_{\Sigma}\right)$ which are adjacent along a subarc of an $\operatorname{arc} \delta \in \mathcal{C}$ that lifts to a boundary arc $\widetilde{\delta}$ of $P$. More 


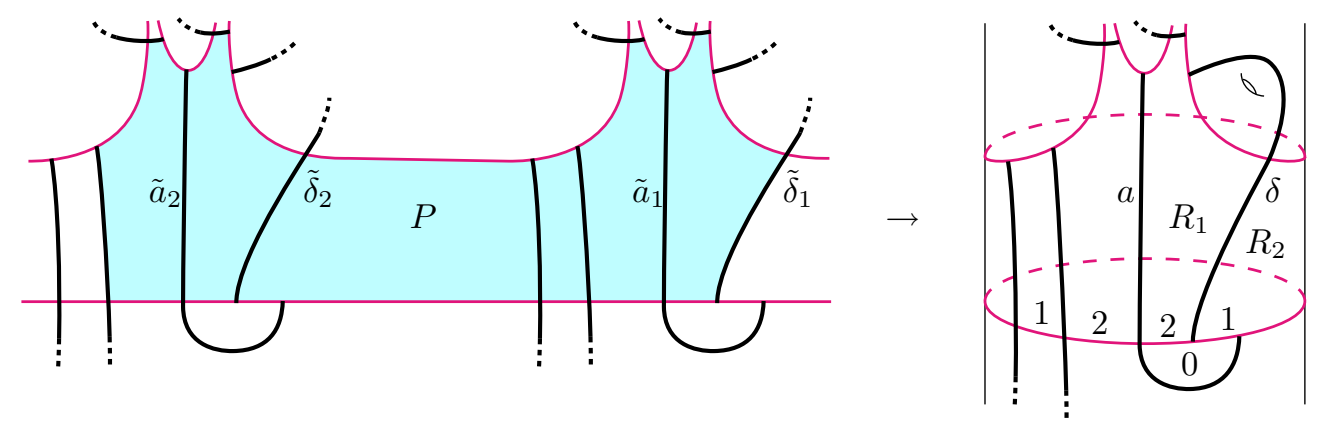

Figure 9: Construction of a virtual pinwheel

precisely, orient $\delta$ so that it starts in $R_{+}$, and denote by $R_{1}$ the region to the left of it and by $R_{2}$ the region to the right (see Figure 9). Then we claim that $w\left(R_{1}\right)>w\left(R_{2}\right)$.

To prove the claim, first note that $R_{1} \neq R_{2}$. If, on the contrary, $R_{1}=R_{2}=R$, then there is an oriented closed curve $\alpha$ such that $\alpha \backslash \delta \subset \operatorname{int}(R)$ which is "dual" to the arc $\delta$ in the sense that they intersect transversely and $\langle\delta, \alpha\rangle=+1$. Observe that any connected component $\widetilde{\alpha}$ of $\pi^{-1}(\alpha) \cap P$ must enter and exit $P$ along components (say $\widetilde{\delta}_{1}$ and $\widetilde{\delta}_{k}$ ) of $\pi^{-1}(\delta)$. Now, the orientation of $\delta$ induces an orientation on the arcs in $\pi^{-1}(\delta)$, and the induced orientation on $\widetilde{\delta}_{1}$ and $\widetilde{\delta}_{k}$ (as seen by intersecting with $\widetilde{\alpha}$ ) is inconsistent with the chirality involved in the definition of a pinwheel.

To see what value $w$ takes on $R_{1}$ and $R_{2}$, let us look at the components $\widetilde{\delta}_{i}, i=1, \ldots, n$, of $\pi^{-1}(\delta)$, and denote by $o(\delta), i(\delta)$ and $b(\delta)$ the number of components that are respectively on the outside, in the interior, or on the boundary of $P$. Every time a component $\widetilde{\delta}_{i}$ of $\pi^{-1}(\delta)$ appears on the boundary of $P$, the minimal subpolygon of $P$ adjacent to $\widetilde{\delta}_{i}$ must project to the region $R_{1}$. Hence $w\left(R_{1}\right)=i(\delta)+b(\delta)$ and $w\left(R_{2}\right)=i(\delta)$, and therefore $w\left(R_{1}\right)>w\left(R_{2}\right)$.

We will now prove that $P^{\prime}$ is a polygon. We first claim that

$$
\pi: \pi^{-1}\left(P^{\prime}\right) \cap P \rightarrow P^{\prime}
$$

is a covering map. Indeed, any subpolygon of $P^{\prime}$ lifts to $\max (w)$ subpolygons of $P$. Moreover, if $a$ is a subarc of an $\operatorname{arc} \delta^{\prime} \in \mathcal{C}$, and $a$ is in $\operatorname{int}\left(P^{\prime}\right)$, then no component $\widetilde{a}_{i}$ of the lift of $a$ can be a side of $P$, since two regions adjacent to it have equal values of $w$. If $U$ is a neighborhood of a point on $a$ in $P^{\prime}$, then $\pi^{-1}(U) \cap P$ consists of $\max (w)$ copies of $U$. Now that we know that $\pi^{-1}\left(P^{\prime}\right) \cap P$ covers $P^{\prime}, P^{\prime}$ must be simply connected, since $\pi^{-1}\left(P^{\prime}\right) \cap P$ must 
be a union of subpolygons of $P^{\prime}$ (hence simply connected), and the cover is a finite cover.

Finally, $\delta \in \mathcal{C}$ which is a side of $P^{\prime}$ and returns to $P^{\prime}$ must enclose a nontrivial element of $\pi_{1}\left(\Sigma, P^{\prime}\right) \simeq \pi_{1}(\Sigma)$; otherwise it cobounds a disk together with $P^{\prime}$, and no cover of $\Sigma$ will extricate the relevant endpoint of $\delta$ from $P^{\prime}$ (and hence $P)$.

Question 3.1 Can we generalize Theorem 1.2 to the case where we have nested bypasses? What's the analogous object to the pinwheel in this case?

\section{Virtual Pinwheels and Tightness}

In this section we will discuss the following question:

Question 4.1 Can we give a necessary and sufficient condition for $(\Sigma, \mathcal{C})$ to be tight, if $\Sigma$ is a convex surface which is either closed or compact with Legendrian boundary?

We will present a partial answer to this question. Before we proceed, we first discuss a useful technique called Bypass Rotation. Let $\Sigma$ be a convex boundary component of a tight contact 3-manifold $(M, \xi)$, and let $\delta_{1}$ and $\delta_{2}$ be disjoint arcs of attachment on $\Sigma$. The bypasses are to be attached from the exterior of $M$, and attached from the front in the figures. Suppose there is an embedded rectangular polygon $R$, where two of the sides are subarcs of $\delta_{1}$ and $\delta_{2}$ and the other two sides are subarcs $\gamma_{1}$ and $\gamma_{2}$ of $\Gamma_{\Sigma}$. Assume $\delta_{1}$ and $\delta_{2}$ both extend beyond $\gamma_{1}$ and do not reintersect $\partial R$. If we position $R, \delta_{1}$, and $\delta_{2}$ as in Figure [10, so that, with the orientation induced from $R, \gamma_{1}$ starts on $\delta_{2}$ and ends on $\delta_{1}$, then we say that $\delta_{1}$ lies to the left of $\delta_{2}$.

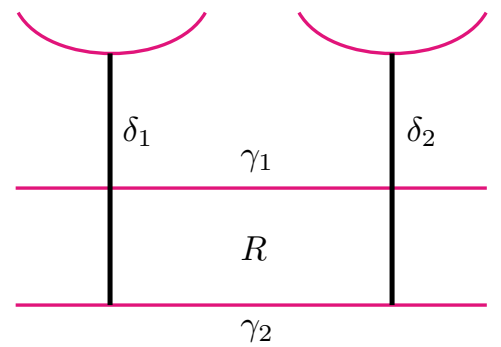

Figure 10: $\delta_{1}$ is to the left of $\delta_{2}$ 
The next lemma shows that the arc of attachment of a bypass can be "rotated to the left" and still preserve tightness. For convenience, let $M\left(\delta_{1}, \ldots, \delta_{k}\right)$ be a contact manifold obtained by attaching $k$ disjoint bypasses from the exterior, along arcs of attachment $\delta_{1}, \ldots, \delta_{k}$.

Lemma 4.2 (Bypass Rotation) Let $(M, \xi)$ be a contact 3 -manifold, and $\delta_{1}, \delta_{2}$ be arcs of attachment on a boundary component $\Sigma$ of $M$. If $\delta_{1}$ is to the left of $\delta_{2}$ and $M\left(\delta_{2}\right)$ is tight, then $M\left(\delta_{1}\right)$ is also tight.

Proof If $M\left(\delta_{2}\right)$, is tight, then $M\left(\delta_{1}, \delta_{2}\right)=\left(M\left(\delta_{2}\right)\right)\left(\delta_{1}\right)$ is also tight, since attaching $\delta_{2}$ makes $\delta_{1}$ a trivial arc of attachment. Now, $M\left(\delta_{1}, \delta_{2}\right)$ is also $\left(M\left(\delta_{1}\right)\right)\left(\delta_{2}\right)$, so $M\left(\delta_{1}\right)$ must be tight.
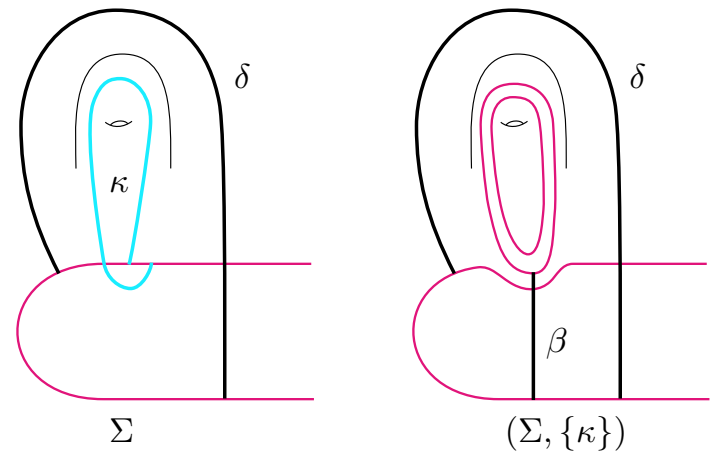

Figure 11: Creating extra dividing curves

It is clear that if there is an actual pinwheel $P$ in $\Sigma$, then $(\Sigma, \mathcal{C})$ is not tight. It is often possible to make the same conclusion in the presence of a virtual pinwheel $P$ by using the technique of Bypass Rotation. In fact, assume that there is a virtual pinwheel $P$ in $\Sigma$ and let $\delta$ be an arc of attachment which encircles a nontrivial element of $\pi_{1}(\Sigma, P)$. Suppose $\delta$ has been oriented so that its orientation coincides with the orientation on $\partial P$. If $\delta$ can be rotated to the left so that the final point of $\delta$ is shifted away from $P$, then the newly obtained configuration contains a pinwheel, and hence $(\Sigma, \mathcal{C})$ is overtwisted by Lemma 4.2. Even if there are no arcs of $\Gamma_{\Sigma}-\partial P$ to which we can rotate $\delta$ without hitting other bypass arcs of attachment, we can often perform a folding operation. This operation can be described in two equivalent ways (see [8] for details): Either add a bypass along the arc $\kappa$ as in Figure 11 to obtain the contact structure $(\Sigma, \mathcal{C} \cup\{\kappa\})$, or fold along a Legendrian divide to create a pair of parallel dividing curves "along" $\delta$. Since both operations can be done inside an invariant neighborhood of $\Sigma,(\Sigma, \mathcal{C} \cup\{\kappa\})$ is tight if $(\Sigma, \mathcal{C})$ is. Now, 
rotating $\delta$ to the left, we can move the endpoint of $\delta$ to be on one of the newly created parallel dividing curves - this yields the bypass $\beta$ pictured in Figure 11 . The configuration $\left(\Sigma, \mathcal{C}^{\prime}\right)$ obtained from $(\Sigma, \mathcal{C} \cup\{\kappa\})$ by replacing $\delta$ by $\beta$ is tight if $(\Sigma, \mathcal{C})$ is tight. However, by repeated application of this procedure if necessary, we will often be able to eventually obtain a genuine pinwheel $P$, hence showing that $(\Sigma, \mathcal{C})$ is overtwisted. More precisely, we have the following:

Proposition 4.3 Let $P$ be a virtual pinwheel in $\Sigma$ and $\delta \in \mathcal{C}$ be an arc of attachment on $\partial P$ which returns to $P$. Decompose $\delta=\delta_{0} \cup \delta_{1}$, where $\delta_{i}$, $i=0,1$, have endpoints on $\Gamma_{\Sigma}$ and $\delta_{0} \subset \partial P$. Orient $\delta$ to agree with the orientation on $\partial P$. Let $Q$ be a connected component of $\Sigma \backslash\left(\Gamma_{\Sigma} \cup\left(\cup_{\beta \in \mathcal{C}} \beta\right)\right)$ so that $\delta_{1} \subset \partial Q$ and the orientation on $\delta_{1}$ agrees with the orientation on $\partial Q$. If one of the following is true for each $\delta$, then $(\Sigma, \mathcal{C})$ is overtwisted:

(1) $Q$ is not a polygon.

(2) $Q$ is a polygon but has sides in $\Gamma_{\Sigma}$ which are not in $\partial P$.

We now consider the situation in which the Bypass Rotation technique just described fails. Let $P$ be a minimal pinwheel, i.e., a pinwheel whose interior does not intersect any arc of attachment in $\mathcal{C}$. Let $\delta$ be an attaching arc of $P$ that returns to $P$ that we cannot "unhook". Then the region $Q$ described in Proposition 4.3 must be polygonal and all of the edges of $Q$ that are coming from the dividing set must be sub-edges of the boundary of $P$. The minimality of $P$ forces edges of $Q$ that are attaching arcs to also be edges of $P$. Thus $Q$ is an anti-pinwheel, that is, a polygon whose edges which are arcs of attachment are oriented in the direction opposite to that of a pinwheel. Two such pinwheel/anti-pinwheel pairs are illustrated in Figure 12.
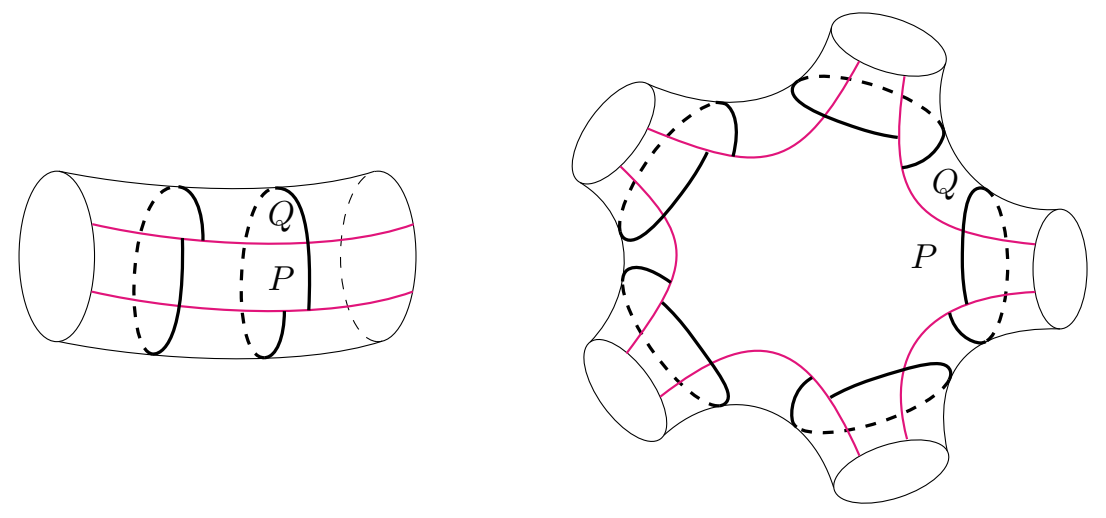

Figure 12: Examples of anti-pinwheels 
Since there is a virtual pinwheel $P$ in the situation described above, $(\Sigma, \mathcal{C})$ is not universally tight by Theorem 1.3 To show that there are cases when $(\Sigma, \mathcal{C})$ is tight but virtually overtwisted, we will analyze the situation indicated in the left portion of Figure 13. Here $\Sigma=T^{2}$, and we think of $\Sigma \times I$ as a neighborhood of the boundary of the solid torus. First, we cut the solid torus along a convex disk $D$ such that its boundary is the curve $\gamma$ that cuts $P \cup Q$ and intersects the dividing set at two points. Next, round the corners that are shown in Figure 13. and we obtain a tight convex ball. In reverse, we can think of the solid torus as obtained by gluing two disks $D_{1}$ and $D_{2}$ on the boundary of $B^{3}$. The bypasses in the picture correspond to adding trivial bypasses to the ball. By applying the basic gluing theorem for gluing across a convex surface with $\partial$-parallel dividing set (see for example Theorem 1.6 in [7]), we see that $(\Sigma, \mathcal{C})$ is tight.

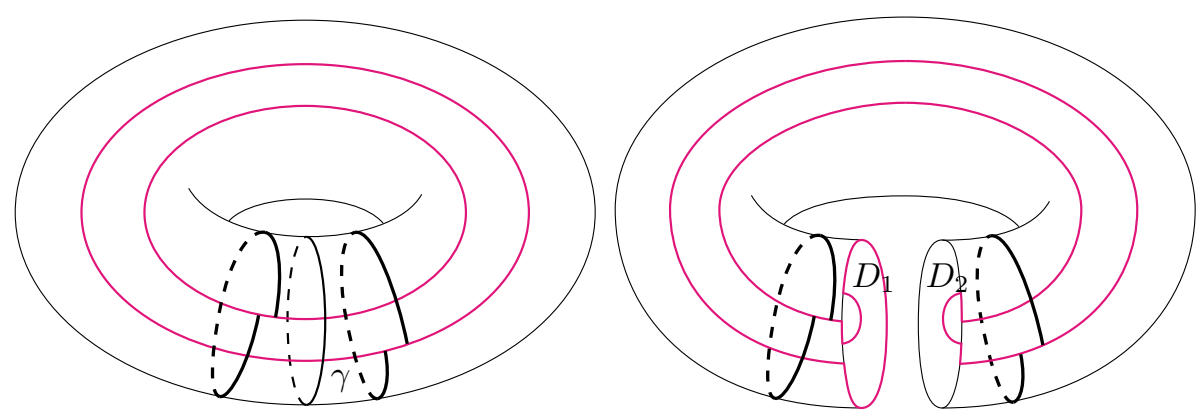

Figure 13: Cutting the pinwheel

Attempts at generalizing the above technique quickly run into some difficulties. Suppose we want to iteratively split $\Sigma$ along a closed curve $\gamma$ and glue in disks $D_{1}$ and $D_{2}$. One difficulty (although not the only one) is that at some step in the iteration we could get an overtwisted structure on $\left(\Sigma^{\prime}, \mathcal{C}\right)$. ( $\Sigma^{\prime}$ could form contractible dividing curves.) On the other hand, this does not necessarily prove that $(\Sigma, \mathcal{C})$ is overtwisted; it merely occurs as a subset of a space with an overtwisted contact structure.

Acknowledgements $\mathrm{KH}$ wholeheartedly thanks the University of Tokyo, the Tokyo Institute of Technology, and especially Prof. Takashi Tsuboi for their hospitality during his stay in Tokyo during Summer-Fall 2003. He was supported by an Alfred P. Sloan Fellowship and an NSF CAREER Award. GM was supported by NSF grants DMS-0072853 and DMS-0410066 and WHK was supported by NSF grants DMS-0073029 and DMS-0406158. The authors also thank the referee for helpful comments. 


\section{References}

[1] Vincent Colin, Une infinité de structures de contact tendues sur les variétés toroïdales, Comment. Math. Helv. 76 (2001) 353-372 MathReview

[2] Yakov Eliashberg, Contact 3-manifolds twenty years since J. Martinet's work, Ann. Inst. Fourier (Grenoble) 42 (1992) 165-192 MathReview

[3] Emmanuel Giroux, Convexité en topologie de contact, Comment. Math. Helv. 66 (1991) 637-677 MathReview

[4] Emmanuel Giroux, Structures de contact sur les variétés fibrées en cercles audessus d'une surface, Comment. Math. Helv. 76 (2001) 218-262 MathReview

[5] Ko Honda, On the classification of tight contact structures. I, Geom. Topol. 4 (2000) 309-368 MathReview

[6] Ko Honda, William H Kazez, Gordana Matić, Tight contact structures and taut foliations, Geom. Topol. 4 (2000) 219-242 MathReview

[7] Ko Honda, William H Kazez, Gordana Matić, Convex decomposition theory, Int. Math. Res. Not. (2002) 55-88 MathReview

[8] Ko Honda, William H Kazez, Gordana Matić, Tight contact structures on fibered hyperbolic 3-manifolds, J. Differential Geom. 64 (2003) 305-358 MathReview

KH: University of Southern California, Los Angeles, CA 90089, USA

and

WHK and GM: University of Georgia, Athens, GA 30602, USA

Email: khonda@math.usc.edu, will@math.uga.edu,

mailtogordana@math.uga.edu

URL: http://almaak.usc.edu/ khonda, http://www.math.uga.edu/ will, http://www . math.uga.edu/ gordana

Received: 21 January 2005 